\title{
Secure and Efficient Protocols for Iris and Fingerprint Identification
}

\author{
Marina Blanton ${ }^{1}$ and Paolo Gasti ${ }^{2}$ \\ 1 Department of Computer Science and Engineering, University of Notre Dame \\ 2 Department of Information and Computer Science, University of California, Irvine
}

\begin{abstract}
Recent advances in biometric recognition and the increasing use of biometric data prompt significant privacy challenges associated with the possible misuse, loss, or theft of biometric data. Biometric matching is often performed by two mutually distrustful parties, one of which holds one biometric image while the other owns a possibly large biometric collection. Due to privacy and liability considerations, neither party is willing to share its data. This gives rise to the need to develop secure computation techniques over biometric data where no information is revealed to the parties except the outcome of the comparison or search. To address the problem, in this work we develop and implement the first privacy-preserving identification protocol for iris codes. We also design and implement a secure protocol for fingerprint identification based on FingerCodes with a substantial improvement in the performance compared to existing solutions. We show that new techniques and optimizations employed in this work allow us to achieve particularly efficient protocols suitable for large data sets and obtain notable performance gain compared to the state-of-the-art prior work.
\end{abstract}

\section{Introduction}

Recent advances in biometric recognition make the use of biometric data more prevalent for authentication and other purposes. Today large-scale collections of biometric data include face, fingerprint, and iris images collected by the US Department of Homeland Security (DHS) from visitors through its US-VISIT program [21], iris images collected by the United Arab Emirates (UAE) Ministry of Interior from all foreigners and also fingerprints and photographs from certain types of travelers [23], and several others. While biometry serves as an excellent mechanism for authentication and identification of individuals, such data is undeniably extremely sensitive and must be well protected. Furthermore, once leaked biometric data cannot be revoked or replaced. For these reasons, biometric data cannot be easily shared between organizations or agencies. However, there could be legitimate reasons to carry out computations on biometric data belonging to different entities. For example, a non-government agency may need to know whether a biometric it possesses appears on the government watch-list. In this case the agency would like to maintain the privacy of the individual if no matches are found, and the government also does not want to release its database to third parties.

The above requires carrying out computation over biometric data in a way that keeps the data private and reveals only the outcome of the computation. In particular, we study 
the problem of biometric identification, where a client $C$ is in a possession of a biometric $X$ and a server $S$ possesses a biometric database $D$. The client would like to know whether $X$ appears in the database $D$ by comparing its biometric to the records in $D$. The computation amounts to comparing $X$ to each $Y \in D$ in a privacy-preserving manner. This formulation is general enough to apply to a number of other scenarios, ranging from a single comparison of $X$ and $Y$ to the case where two parties need to compute the intersection of their respective databases. We assume that the result of comparing biometrics $X$ and $Y$ is a bit, and no additional information about $X$ or $Y$ should be learned by the parties as a result of secure computation. With our secure protocols, the outcome can be made available to either party or both of them; for concreteness in our description, we have the client learn the outcome of each comparison.

In this work we assume that both the client's and the server's biometric images have been processed and have representations suitable for biometric matching, i.e., each raw biometric image has been processed by a feature extraction algorithm. For the types of biometric considered in this work, this can be performed for each image independently and we do not discuss this further.

Prior Work. Literature on secure multi-party computation is extensive. Starting from the seminal work on garbled circuit evaluation [39], it has been known that any function can be securely evaluated by representing it as a boolean circuit. Similar results are also known for securely evaluating any function using secret sharing techniques (e.g., [36]) or homomorphic encryption (e.g., [11]). In the last several years a number of tools have been developed for automatically creating a secure protocol from a function description written in a high-level language. Examples include Fairplay [30], VIFF [14], TASTY [18], and others. It is, however, well-known that custom optimized protocols are often constructed for specific applications due to the inefficiency of generation solution. Such custom solutions are known for a wide range of application (e.g., set operations [29]17], DNA matching [38], k-means clustering [9], etc.), and this work focuses on secure biometric identification using iris codes and fingerprints. Furthermore, some of the optimizations employed in this work can find their uses in protocol design for other applications, as well as general compilers and tools such as TASTY [18].

With the growing prevalence of applications that use biometrics, the need for secure biometric identification was recognized in the research community. A number of recent publications address the problem of privacy-preserving face recognition [163733]. This problem was first treated by Erkin et al. [16], where the authors designed a privacypreserving face recognition protocol based on the Eigenfaces algorithm. The performance of that solution was consequently improved by Sadeghi et al. [37]. More recently, Osadchy et al. [33] designed a new face recognition algorithm together with its privacy-preserving realization called SCiFI. The design targeted to simultaneously address robustness to different viewing conditions and efficiency when used for secure computation. As a result, SCiFI is currently recognized as the best face identification algorithm with efficient privacy-preserving realization. SCiFI takes $0.31 \mathrm{sec}$ (during the online phase) [33] to compare two biometrics, and therefore would take about $99 \mathrm{sec}$ to compare a biometric to a database of 320 images (which is the database size used in the experiments in several prior publications). 
Another very recent work by Barni et al. [3] designs a privacy-preserving protocol for fingerprint identification using FingerCodes [25]. FingerCodes use texture information from a fingerprint to compare two biometrics. The algorithm is not as discriminative as fingerprint matching techniques based on location of minutiae points, but it was chosen by the authors as particularly suited for efficient realization in the privacy-preserving framework. As of the time of this writing, similar results for other types of biometrics or other fingerprint matching techniques are not available in the literature. We narrow this gap by providing a secure two-party protocol for widely used iris identification, as well as address fingerprint identification. Our protocols follow the standard algorithms for comparing two biometrics, yet they are very efficient and outperform the state-ofthe-art protocols with a notable reduction in the overhead.

Bringer et al. [8] describe a biometric-based authentication mechanism with privacy protection of biometric, where the Hamming distance is used as the distance metric. The authentication server is composed of three entities that must not collude, and one of them, the matcher, learns the computed Hamming distance. In our work, however, no information beyond the outcome of the comparison is revealed, the computation itself is more complex and corresponds to the actual algorithm used for iris code comparisons, and there is no need for additional or third-party entities. Barbosa et al. [2] extend the framework with a classifier to improve authentication accuracy and propose an instantiation based on Support Vector Machine using homomorphic encryption.

Our Contributions. In this work we treat the problem of privacy preserving biometric identification. We develop new secure protocols for two types of biometric, iris and fingerprints, and achieve security against semi-honest adversaries. While iris codes are normally represented as binary strings and use very similar matching algorithms, there is a variety of representations and comparison algorithms for fingerprints. In this paper, we study FingerCodes that use fixed-size representations and an efficient comparison algorithm.11 Our protocols were designed with efficiency in mind to permit their use on relatively large databases, and possibly in real time. While direct performance comparison of our protocols and the results available in the literature is possible only in the case of FingerCode, we can use complexity of the computation to draw certain conclusions. The results we achieve in this work are as follows:

1. Our secure FingerCode protocol is extremely fast and allows the parties to compare two fingerprints $X$ and $Y$ using a small fraction of a second. For a database of 320 biometrics, the online computation can be carried out in $0.45 \mathrm{sec}$ with the communication of $279 \mathrm{~KB}$. This is an over 30 -fold improvement in both communication and computation over the privacy-preserving solution of [3], as detailed in Section 5] and a significant improvement over an adaptation of [37] to this context.

2. Iris codes use significantly longer representations (thousands of bits) and require more complex transformation of the data. Despite the length and complexity, our solution allows two iris codes to be compared in $0.15 \mathrm{sec}$. With respect to the stateof-the-art face recognition protocol SCiFI, which also relies on Hamming distance

\footnotetext{
${ }^{1}$ We also construct a secure protocol for minutiae-based fingerprint comparisons, but its description and implementation appear in the full version of this work [7] due to space constraints.
} 
computation, our protocol achieves lower overhead despite the fact that the computation involves an order of magnitude larger number of more complex operations.

\section{Description of Computation}

In what follows, we assume that client $C$ holds a single biometric $X$ and server $S$ holds a database of biometrics $D$. The goal is to learn whether $C$ 's biometric appears in $S$ 's database without learning any additional information. This is accomplished by comparing $X$ to each biometric $Y \in D$, and as a result of each comparison $C$ learns a bit that indicates whether the comparison resulted in a match.

Iris. Let an iris biometric $X$ be represented as an $m$-bit binary string. We use $X_{i}$ to denote $i$-th bit of $X$. In iris-based recognition, after feature extraction, biometric matching is normally performed by computing the Hamming distance between two biometric representations. Furthermore, the feature extraction process is such that some bits of the extracted string $X$ are unreliable and are ignored in the matching process. Information about such bits is stored in an additional $m$-bit string, called mask, where its $i$-th bit is set to 1 if the $i$-th bit of $X$ should be used in the matching process and is set to 0 otherwise. For biometric $X$, we use $M(X)$ to denote the mask associated with $X$. Often, a predetermined number of bits (e.g., 25\% in [20] and 35\% in [4]) is considered unreliable in each biometric template. Thus, to compare two biometric representations $X$ and $Y$, their Hamming distance takes into account the respective masks. That is, if the Hamming distance between two iris codes without masks is computed as: $H D(X, Y)=(\|X \oplus Y\|) / m=\left(\sum_{i=1}^{m} X_{i} \oplus Y_{i}\right) / m$, the computation of the Hamming distance that uses masks becomes [15]:

$$
H D(X, M(X), Y, M(Y))=\frac{\|(X \oplus Y) \cap M(X) \cap M(Y)\|}{\|M(X) \cap M(Y)\|}
$$

In other words, we have $H D(X, M(X), Y, M(Y))=\frac{\sum_{i=1}^{m}\left(\left(X_{i} \oplus Y_{i}\right) \wedge M\left(X_{i}\right) \wedge M\left(Y_{i}\right)\right)}{\sum_{i=1}^{m}\left(M\left(X_{i}\right) \wedge M\left(Y_{i}\right)\right)}$. Throughout this work, we assume that the latter formula is used and simplify the notation to $H D(X, Y)$. Then the computed Hamming distance is compared with a specific threshold $T$, and the biometrics $X$ and $Y$ are considered to be a match if the distance is below the threshold, and a mismatch otherwise. The threshold $T$ is chosen based on the distributions of authentic and impostor data. (In the likely case of overlap of the two distributions, the threshold is set to achieve the desired levels of false accept and false reject rates based on the security goals.)

Two iris representations can be slightly misaligned. This problem is caused by head tilt during image acquisition. To account for this, the matching process attempts to compensate for the error and rotates the biometric representation by a fixed amount to determine the lowest distance. Each biometric is represented as a two-dimensional array, therefore a circular shift is applied to each row by shifting its representation by a small fixed number of times, which we denote by $c$. The minimum Hamming distance across all runs is then compared to the threshold. That is, if we let $\operatorname{LS}^{j}(\cdot)$ (resp., $\left.\mathrm{RS}^{j}(\cdot)\right)$ denote a circular left (resp., right) shift of the argument by a fixed number of bits $(2$ bits in experiments conducted by the biometrics group at our institution, where 
application of the Gabor filter during feature extraction results in a complex number, which is quantized into a 2-bit value), the matching process becomes:

$$
\begin{array}{r}
\min \left(H D\left(X, \operatorname{LS}^{c}(Y)\right), \ldots, H D\left(X, \operatorname{LS}^{1}(Y)\right), H D(X, Y),\right. \\
\left.H D\left(X, \operatorname{RS}^{1}(Y)\right), \ldots, H D\left(X, \operatorname{RS}^{c}(Y)\right)\right) \stackrel{?}{<} T
\end{array}
$$

Throughout this work we assume that the algorithms for comparing two biometrics are public, as well as any constant parameters such as $T$. Our protocols, however, maintain their security and performance guarantees if the (fixed) thresholds are known only to the server who owns the database.

Fingerprints. Work on fingerprint identification dates many years back with a number of different approaches currently available (see, e.g., [31] for an overview). The most popular and widely used techniques extract information about minutiae from a fingerprint and store that information as a set of points in the two-dimensional plane. Fingerprint matching can also be performed using a different type of information extracted from a fingerprint image. One example is FingerCode [25] which uses texture information from a fingerprint scan to form fingerprint representation $X$. While FingerCodes are not as distinctive as minutiae-based representations and are best suited for use in combination with minutiae to improve the overall matching accuracy [31], FingerCodebased identification can be implemented very efficiently in a privacy-preserving protocol. In particular, each FingerCode consists of a fixed number $m$ elements of $\ell$ bits each. Then FingerCodes $X=\left(x_{1}, \ldots, x_{m}\right)$ and $Y=\left(y_{1}, \ldots, y_{m}\right)$ are considered a match if the Euclidean distance between their elements is below the threshold $T$ :

$$
\sqrt{\sum_{i=1}^{m}\left(x_{i}-y_{i}\right)^{2}} \stackrel{?}{<} T
$$

Barni et al. [3] was the first to provide a privacy-preserving protocol for FingerCodebased biometric identification. We show that the techniques employed in this work improve both computation and communication of the protocol of [3] by a large factor.

\section{Preliminaries}

Security Model. We use the standard security model for secure two-party computation in presence of semi-honest participants (also known as honest-but-curious or passive). In particular, it means that the parties follow the prescribed behavior, but might try to compute additional information from the information obtained during protocol execution. Security in this setting is defined using simulation argument: the protocol is secure if the view of protocol execution for each party is computationally indistinguishable from the view simulated using that party's input and output only. This means that the protocol execution does not reveal any additional information to the participants. The definition below formalizes the notion of security for semi-honest participants:

Definition 1. Let parties $P_{1}$ and $P_{2}$ engage in a protocol $\pi$ that computes function $f\left(\mathrm{in}_{1}, \mathrm{in}_{2}\right)=\left(\right.$ out $_{1}$, out $\left._{2}\right)$, where $\mathrm{in}_{i}$ and out ${ }_{i}$ denote input and output of party $P_{i}$, respectively. Let $\mathrm{VIEW}_{\pi}\left(P_{i}\right)$ denote the view of participant $P_{i}$ during the execution 
of protocol $\pi$. More precisely, $P_{i}$ 's view is formed by its input, internal random coin tosses $r_{i}$, and messages $m_{1}, \ldots, m_{t}$ passed between the parties during protocol execution, i.e., $\operatorname{VIEW}_{\pi}\left(P_{i}\right)=\left(\mathrm{in}_{i}, r_{i}, m_{1}, \ldots, m_{t}\right)$. We say that protocol $\pi$ is secure against semi-honest adversaries if for each party $P_{i}$ there exists a probabilistic polynomial time simulator $S_{i}$ such that $\left\{S_{i}\left(\mathrm{in}_{i}, f\left(\mathrm{in}_{1}, \mathrm{in}_{2}\right)\right)\right\} \equiv\left\{\operatorname{VIEW}_{\pi}\left(P_{i}\right)\right.$, out $\left.{ }_{i}\right\}$, where “三” denotes computational indistinguishability.

Homomorphic Encryption. Our constructions use a semantically secure additively homomorphic encryption scheme. In an additively homomorphic encryption scheme, $\operatorname{Enc}\left(m_{1}\right) \cdot \operatorname{Enc}\left(m_{2}\right)=\operatorname{Enc}\left(m_{1}+m_{2}\right)$ which also implies that $\operatorname{Enc}(m)^{a}=\operatorname{Enc}(a \cdot m)$. While any encryption scheme with the above properties (such as the well known Paillier encryption scheme [34]) suffices for the purposes of this work, the construction due to Damgård et al. [13 12] (DGK) is of particular interest here. We also note that in Paillier encryption scheme, a public key consists of a $k$-bit RSA modulus $N=p q$, where $p$ and $q$ are prime, and an element $g$ whose order is a multiple of $N$ in $\mathbb{Z}_{N^{2}}^{*}$. Given a message $m \in \mathbb{Z}_{N}$, encryption is performed as $\operatorname{Enc}(m)=g^{m} r^{n} \bmod N^{2}$, where $r \stackrel{R}{\leftarrow} \mathbb{Z}_{N}$ and notation $a \stackrel{R}{\leftarrow} A$ means that $a$ is chosen uniformly at random from the set $A$. In DGK encryption scheme [13 12], which was designed to work with small plaintext spaces and has shorter ciphertext size than other randomized encryption schemes, a public key consists of (i) a (small, possibly prime) integer $u$ that defines the plaintext space, (ii) $k$-bit RSA modulus $N=p q$ such that $p$ and $q$ are $k / 2$-bit primes, $v_{p}$ and $v_{q}$ are $t$-bit primes, and $u v_{p} \mid(p-1)$ and $u v_{q} \mid(q-1)$, and (iii) elements $g, h \in \mathbb{Z}_{N}^{*}$ such that $g$ has order $u v_{p} v_{q}$ and $h$ has order $v_{p} v_{q}$. Given a message $m \in \mathbb{Z}_{u}$, encryption is performed as $\operatorname{Enc}(m)=g^{m} h^{r} \bmod N$, where $r \stackrel{R}{\leftarrow}\{0,1\}^{2.5 t}$. We refer the reader to the original publications [34] and [13[12], respectively, for any additional information.

Garbled Circuit Evaluation. Originated in Yao's work [39], garbled circuit evaluation allows two parties to securely evaluate any function represented as a boolean circuit. The basic idea is that, given a circuit composed of gates, one party $P_{1}$ creates a garbled circuit by assigning to each wire two randomly chosen keys. $P_{1}$ also encodes gate information in a way that given keys corresponding to the input wires (encoding specific inputs), the key corresponding to the output of the gate on those inputs can be recovered. The second party, $P_{2}$, evaluates the circuit using keys corresponding to inputs of both $P_{1}$ and $P_{2}$ (without learning anything in the process). At the end, the result of the computation can be recovered by linking the output keys to the bits which they encode.

Recent literature provides optimizations that reduce computation and communication overhead associated with circuit construction and evaluation. Kolesnikov and Schneider [27] describe an optimization that permits XOR gates to be evaluated for free, i.e., there is no communication overhead associated with such gates and their evaluation does no involve cryptographic functions. This optimization is possible when the hash function used for creating garbled gates can be assumed to be correlation robust (see [28 27] for more detail). Under the same assumptions, Pinkas et al. [35] additionally give a mechanism for reducing communication complexity of binary gates by $25 \%$ : now each gate can be specified by encoding only three outcomes of the gate instead of all four. Finally, Kolesnikov et al. [26] improve the complexity of certain commonly 
used operations such as addition, multiplication, comparison, etc. by reducing the number of non-XOR gates: adding two $n$-bit integers requires $5 n$ gates, $n$ of which are non-XOR gates; comparing two $n$-bit integers requires $4 n$ gates, $n$ of which are nonXOR gates; and computing the minimum of $t n$-bit integers (without the location of the minimum value) requires $7 n(t-1)$ gates, $2 n(t-1)$ of which are non-XOR gates.

With the above techniques, evaluating a non-XOR gates involves one invocation of the hash function (which is assumed to be correlation robust). During garbled circuit evaluation, $P_{2}$ directly obtains keys corresponding to $P_{1}$ 's inputs from $P_{1}$ and engages in the oblivious transfer (OT) protocol to obtain keys corresponding to $P_{2}$ 's inputs.

Oblivious Transfer. In 1-out-of-2 Oblivious Transfer, $O T_{1}^{2}$, one party, the sender, has as its input two strings $m_{0}, m_{1}$ and another party, the receiver, has as its input a bit $b$. At the end of the protocol, the receiver learns $m_{b}$ and the sender learns nothing. Similarly, in 1-out-of- $N$ OT the receiver obtains one of the $N$ strings held by the sender. There is a rich body of research literature on OT, and in this work we use its efficient implementation from [32] as well as techniques from [24] that reduce a large number of OT protocol executions to $\kappa$ of them, where $\kappa$ is the security parameter. This, in particular, means that obtaining the keys corresponding to $P_{2}$ 's inputs in garbled circuit evaluation by $P_{2}$ incurs only small overhead.

\section{Secure Iris Identification}

As indicated in equation 1 computing the distance between two iris codes involves performing the division operation. While techniques for carrying out this operation using secure multi-party computation are known (see, e.g., [19610]), their performance in practice even using very recent results is far from satisfactory for this application As an example, Blanton [5] reports that two-party evaluation of garbled circuits produced by Fairplay takes several seconds for numbers of length 24-28 bits, but circuits for longer integers could not be constructed due to the rapidly increasing memory requirements of Fairplay. Hoens et al. [19] report that building a multi-party division protocol using homomorphic encryption alone requires on the order of an hour to carry out the operation for 32-bit integers. Fortunately, in our case the computation can be rewritten to completely avoid this operation and replace it with multiplication. That is, using the notation $H D(X, Y)=\|(X \oplus Y) \cap M(X) \cap M(Y)\| /\|M(X) \cap M(Y)\|=$ $D(X, Y) / M(X, Y)$, instead of testing whether $H D(X, Y) \stackrel{?}{<} T$, we can test whether $D(X, Y) \stackrel{?}{<} T \cdot M(X, Y)$. While the computation of the minimum distance as used in equation 2 is no longer possible, we can replace it with equivalent computation that does not increase its cost. Now the computation becomes:

$$
\left(D\left(X, \operatorname{LS}^{c}(Y)\right) \stackrel{?}{<} T \cdot M\left(X, \operatorname{LS}^{c}(Y)\right)\right) \vee \cdots \vee\left(D\left(X, \operatorname{RS}^{c}(Y)\right) \stackrel{?}{<} T \cdot M\left(X, \operatorname{RS}^{c}(Y)\right)\right)
$$

When this computation is carried over real numbers, $T$ lies in the range $[0,1]$. In our case, we need to carry the computation over the integers, which means that we "scale up" all values with the desired level of precision. That is, by using $\ell$ bits to achieve 
desired precision, we multiply $D(X, Y)$ by $2^{\ell}$ and let $T$ range between 0 and $2^{\ell}$. Now $2^{\ell} D(X, Y)$ and $T \cdot M(X, Y)$ can be represented using $\lceil\log m\rceil+\ell$ bits.

Security. Due to space constraints, we defer the security analysis of our iris identification protocol, described in Sections 4.1 and 4.2 below, to Appendix A

\subsection{Base Protocol}

In what follows, we first describe the protocol in its simplest form. Section 4.2 presents optimizations and the resulting performance of the protocol.

In our solution, the client $C$ generates a public-private key pair $(p k, s k)$ for a homomorphic encryption scheme and distributes the public key $p k$. This is a one-time setup cost for the client for all possible invocations of this protocol with any number of servers. During the protocol itself, the secure computation proceeds as specified in equation 4 . In the beginning, $C$ sends its inputs encrypted with $p k$ to the server $S$. At the server side, the computation first proceeds using homomorphic encryption, but later the client and the server convert the intermediate result into a split form and finish the computation using garbled circuit evaluation. This is due to the fact that secure twoparty computation of the comparison is the fastest using garbled circuit evaluation [26], but the rest of the computation in our case is best performed on encrypted values.

To compute $D(X, Y)=\sum_{i=1}^{m}\left(X_{i} \oplus Y_{i}\right) \wedge M\left(X_{i}\right) \wedge M\left(Y_{i}\right)$ using algebraic computation, we use $X_{i} \oplus Y_{i}=X_{i}\left(1-Y_{i}\right)+\left(1-X_{i}\right) Y_{i}$ and obtain $D(X, Y)=\sum_{i=1}^{m}\left(X_{i}(1-\right.$ $\left.\left.Y_{i}\right)+\left(1-X_{i}\right) Y_{i}\right) M\left(X_{i}\right) M\left(Y_{i}\right) . M(X, Y)$ is computed as $\sum_{i=1}^{m} M\left(X_{i}\right) M\left(Y_{i}\right)$. Then if $S$ obtains encryptions of $X_{i} M\left(X_{i}\right),\left(1-X_{i}\right) M\left(X_{i}\right)$, and $M\left(X_{i}\right)$ for each $i$ from $C$, the server will be able to compute $D(X, Y)$ and $M(X, Y)$ using its knowledge of the $Y_{i}$ 's and the homomorphic properties of the encryption. Figure 1 describes the protocol, in which after receiving $C$ 's encrypted values $S$ produces $\operatorname{Enc}\left(M\left(X_{i}\right)\right)$ 's and proceeds to compute $D\left(X, Y^{j}\right)$ and $M\left(X, Y^{j}\right)$ in parallel for each $Y$ in its database, where $Y^{j}$ denotes biometric $Y$ shifted by $j$ positions and $j$ ranges from $-c$ to $c$. At the end of steps 3(a).i and 3(a).ii the server obtains $\operatorname{Enc}\left(2^{\ell} D\left(X, Y^{j}\right)+r_{S}^{j}\right)$ for a randomly chosen $r_{S}^{j}$ of its choice, and at the end of step 3(a).iii $S$ obtains $\operatorname{Enc}\left(T \cdot M\left(X, Y^{j}\right)+t_{S}^{j}\right)$ for a random $t_{S}^{j}$ of its choice. The server sends these values to the client who decrypt them. Therefore, at the end of step 3(a) $C$ holds $r_{C}^{j}=2^{\ell} D\left(X, Y^{j}\right)+r_{S}^{j}$ and $t_{C}^{j}=T \cdot M\left(X, Y^{j}\right)+t_{S}^{j}$ and $S$ holds $-r_{S}^{j}$ and $-t_{C}^{j}$, i.e., they additively share $2^{\ell} D\left(X, Y^{j}\right)$ and $T \cdot M\left(X, Y^{j}\right)$.

What remains to compute is $2 c+1$ comparisons (one per each $Y^{j}$ ) followed by $2 c$ OR operations as specified by equation 4. This is accomplished using garbled circuit evaluation, where $C$ enters $r_{C}^{j}$ 's and $t_{C}^{j}$ 's and $S$ enters $r_{S}^{j}$ 's and $t_{S}^{j}$ 's and they learn a bit, which indicates whether $Y$ was a match.

Note that since $r_{C}^{j}$ 's, $r_{S}^{j}$ 's, $t_{C}^{j}$ 's and $t_{S}^{j}$ 's are used as inputs to the garbled circuit and will need to be added inside the circuit, we want them to be as small as possible. Therefore, instead of providing unconditional hiding by choosing $t_{S}^{j}$ and $r_{C}^{j}$ from $\mathbb{Z}_{N}^{*}$ (where $N$ is from $p k$ ), the protocol achieves statistical hiding by choosing these random values to be $\kappa$ bits longer than the values that they protect, where $\kappa$ is a security parameter. 
Input: $C$ has biometric $X, M(X)$ and key pair $(p k, s k) ; S$ has a database $D$ composed of $Y$, $M(Y)$ biometrics.

Output: $C$ learns what records in $D$ resulted in match with $X$ if any, i.e., it learns a bit as a result of comparison of $X$ with each $Y \in D$.

Protocol steps:

1. For each $i=1, \ldots, m, C$ computes encryptions $\left\langle a_{i 1}, a_{i 2}\right\rangle=\left\langle\operatorname{Enc}\left(X_{i} M\left(X_{i}\right)\right)\right.$, $\operatorname{Enc}((1-$ $\left.\left.\left.X_{i}\right) M\left(X_{i}\right)\right)\right\rangle$ and sends them to $S$.

2. For each $i=1, \ldots, m, S$ computes encryption of $M\left(X_{i}\right)$ by setting $a_{i 3}=a_{i 1} \cdot a_{i 2}=$ $\operatorname{Enc}\left(X_{i} M\left(X_{i}\right)\right) \cdot \operatorname{Enc}\left(\left(1-X_{i}\right) M\left(X_{i}\right)\right)=\operatorname{Enc}\left(M\left(X_{i}\right)\right)$.

3. For each record $Y$ in the database, $S$ and $C$ perform the following steps in parallel:

(a) For each amount of shift $j=-c, \ldots, 0, \ldots, c, S$ rotates the bits of $Y$ by the appropriate number of positions to obtain $Y^{j}$ and proceeds with all $Y^{j}$,s in parallel.

i. To compute $\left(X_{i} \oplus Y_{i}^{j}\right) M\left(X_{i}\right) M\left(Y_{i}^{j}\right)=\left(X_{i}\left(1-Y_{i}^{j}\right)+(1-\right.$ $\left.\left.X_{i}\right) Y_{i}^{j}\right) M\left(X_{i}\right) M\left(Y_{i}^{j}\right)$ in encrypted form, $S$ computes $b_{i}^{j}=a_{i 1}^{\left(1-Y_{i}^{j}\right) M\left(Y_{i}^{j}\right)}$. $a_{i 2}^{Y_{i}^{j} M\left(Y_{i}^{j}\right)}=\operatorname{Enc}\left(X_{i} M\left(X_{i}\right)\left(1-Y_{i}^{j}\right) M\left(Y_{i}^{j}\right)+\left(1-X_{i}\right) M\left(X_{i}\right) Y_{i}^{j} M\left(Y_{i}^{j}\right)\right)$. ii. $S$ adds the values contained in $b_{i}^{j}$,s to obtain $b^{j}=\prod_{i=1}^{m} b_{i}^{j}=\operatorname{Enc}\left(\sum_{i=1}^{m}\left(X_{i} \oplus\right.\right.$ $\left.\left.Y_{i}^{j}\right) M\left(X_{i}\right) M\left(Y_{i}^{j}\right)\right)=\operatorname{Enc}\left(\left\|\left(X \oplus Y^{j}\right) \cap M(X) \cap M\left(Y^{j}\right)\right\|\right) . S$ then "lifts up" the result, blinds, and randomizes it as $c^{j}=\left(b^{j}\right)^{2^{\ell}}$. Enc $\left(r_{S}^{j}\right)$, where $r_{S}^{j} \stackrel{R}{\leftarrow}$ $\{0,1\}^{\lceil\log m\rceil+\ell+\kappa}$, and sends the resulting $c^{j}$ to $C$.

iii. To obtain $T\left(\left\|M(X) \cap M\left(Y^{j}\right)\right\|\right), S$ computes $d_{i}^{j}=a_{i 3}^{M\left(Y_{i}^{j}\right)}=\operatorname{Enc}\left(M\left(X_{i}\right)\right.$. $\left.M\left(Y_{i}^{j}\right)\right)$ and $d^{j}=\left(\prod_{i=1}^{m} d_{i}^{j}\right)^{T}=\operatorname{Enc}\left(T\left(\sum_{i=1}^{m} M\left(X_{i}\right) M\left(Y_{i}^{j}\right)\right)\right) . S$ blinds and randomizes the result as $e^{j}=d^{j} \cdot \operatorname{Enc}\left(t_{S}^{j}\right)$, where $t_{S}^{j} \stackrel{R}{\leftarrow}\{0,1\}^{\lceil\log m\rceil+\ell+\kappa}$, and sends $e^{j}$ to $C$.

iv. $C$ decrypts the received values and sets $r_{C}^{j}=\operatorname{Dec}\left(c^{j}\right)$ and $t_{C}^{j}=\operatorname{Dec}\left(e^{j}\right)$.

(b) $C$ and $S$ perform $2 c+1$ comparisons and OR of the results of the comparisons using garbled circuit. $C$ enters $r_{C}^{j}$ 's and $t_{C}^{j}$ 's, $S$ enters $-r_{S}^{j}$ 's and $-t_{S}^{j}$ 's, and $C$ learns bit $b$ computed as $\bigvee_{j=-c}^{c}\left(\left(r_{C}^{j}-r_{S}^{j}\right) \stackrel{?}{<}\left(t_{C}^{j}-t_{S}^{j}\right)\right)$. To achieve this, $S$ creates the garbled circuit and sends it to $C$. $C$ obtains keys corresponding to its inputs using OT, evaluates the circuit, and $S$ sends to $C$ the key-value mapping for the output gate.

Fig. 1. Secure two-party protocol for iris identification

\subsection{Optimizations}

Pre-computation and Offline Communication. Similar to prior literature on secure biometric identification [16]37|33]3], we distinguish between offline and online stages, where any computation and computation that does not depend on the inputs of the participating parties can be moved to the offline stage. In our protocol, first notice that most modular exponentiations (the most expensive operation in the encryption scheme) can be precomputed. That is, the client needs to produce $2 m$ encryptions of bits. Because both $m$ and the average number of 0's and 1's in a biometric and a mask are known, the client can produce a sufficient number of bit encryptions in advance. In particular, $X$ normally will have $50 \%$ of 0 's and $50 \%$ of 1 's, while $75 \%$ (or a similar number) of $M(X)$ 's bits are set to 1 and $25 \%$ to 0 during biometric processing. Let $p_{0}$ and $p_{1}\left(q_{0}\right.$ and $\left.q_{1}\right)$ denote the fraction of 0 's and 1 's in 
an iris code (resp., its mask), where $p_{0}+p_{1}=q_{0}+q_{1}=1$. Therefore, to have a sufficient supply of ciphertexts to form tuples $\left\langle a_{i 1}, a_{i 2}\right\rangle$, the client needs to precompute $\left(2 q_{0}+q_{1}\left(p_{1}+\varepsilon\right)+q_{1}\left(p_{0}+\varepsilon\right)\right) m=\left(1+q_{0}+2 q_{1} \varepsilon\right) m$ encryptions of 0 and $\left(q_{1}\left(p_{1}+\varepsilon\right)+q_{1}\left(p_{0}+\varepsilon\right)\right) m=q_{1}(1+2 \varepsilon) m$ encryptions of 1 , where $\varepsilon$ is used as a cushion since the number of 0 's and 1 's in $X$ might not be exactly $p_{0}$ and $p_{1}$, respectively. Then at the time of the protocol the client simply uses the appropriate ciphertexts to form its transmission.

Similarly, the server can precompute a sufficient supply of encryptions of $r_{S}^{j}$ 's and $t_{S}^{j}$ 's for all records. That is, the server needs for produce $2(2 c+1)|D|$ encryptions of different random values of length $\lceil\log m\rceil+\ell+\kappa$, where $|D|$ denotes the size of the database $D$. The server also generates one garbled circuit per record $Y$ in its database (for step 3(b) of the protocol) and communicates the circuits to the client. In addition, the most expensive part of the oblivious transfer can also be performed during the offline stage, as detailed below.

Optimized Multiplication. Server's computation in steps 3(a).i and 3(a).iii of the protocol can be significantly lowered as follows. To compute ciphertexts $b_{i}^{j}, S$ needs to calculate $a_{i 1}^{\left(1-Y_{i}^{j}\right) M\left(Y_{i}^{j}\right)} \cdot a_{i 2}^{Y_{i}^{j} M\left(Y_{i}^{j}\right)}$. Since the bits $Y_{i}^{j}$ and $M\left(Y_{i}^{j}\right)$ are known to $S$, this computation can be rewritten using one of the following cases:

- $Y_{i}^{j}=0$ and $M\left(Y_{i}^{j}\right)=0$ : in this case both $\left(1-Y_{i}^{j}\right) M\left(Y_{i}^{j}\right)$ and $Y_{i}^{j} M\left(Y_{i}^{j}\right)$ are zero, which means that $b_{i}^{j}$ should correspond to an encryption of 0 regardless of $a_{i 1}$ and $a_{i 2}$. Instead of having $S$ create an encryption 0 , we set $b_{i}^{j}$ to the empty value, i.e., it is not used in the computation of $b^{j}$ in step 3(a).ii.

- $Y_{i}^{j}=1$ and $M\left(Y_{i}^{j}\right)=0$ : the same as above.

- $Y_{i}^{j}=0$ and $M\left(Y_{i}^{j}\right)=1$ : in this case $\left(1-Y_{i}^{j}\right) M\left(Y_{i}^{j}\right)=1$ and $Y_{i}^{j} M\left(Y_{i}^{j}\right)=0$, which means that $S$ sets $b_{i}^{j}=a_{i 1}$.

- $Y_{i}^{j}=1$ and $M\left(Y_{i}^{j}\right)=1$ : in this case $\left(1-Y_{i}^{j}\right) M\left(Y_{i}^{j}\right)=0$ and $Y_{i}^{j} M\left(Y_{i}^{j}\right)=1$, and $S$ therefore sets $b_{i}^{j}=a_{i 2}$.

The above implies that only $q_{1} m$ ciphertexts $b_{i}^{j}$ need to be added in step 3(a).ii to form $b^{j}$ (i.e., $q_{1} m-1$ modular multiplications to compute the hamming distance between $m$-element strings).

Similar optimization applies to the computation of $d_{i}^{j}$ and $d^{j}$ in step 3(a).iii of the protocol. That is, when $M\left(Y_{i}^{j}\right)=0, d_{i}^{j}$ is set to the empty value and is not used in the computation of $d^{j}$; when $M\left(Y_{i}^{j}\right)=1, S$ sets $d_{i}^{j}=a_{i 3}$. Consequently, $q_{1} m$ ciphertexts are used in computing $d^{j}$.

To further reduce the number of modular multiplications, we can adopt the idea from [33], which consists of precomputing all possible combinations for ciphertexts at positions $i$ and $i+1$ and reducing the number of modular multiplications used during processing a database record in half. In our case, the value of $b_{i}^{j} b_{i+1}^{j}$ requires computation only when $M\left(Y_{i}^{j}\right)=M\left(Y_{i+1}^{j}\right)=1$. In this case, computing $a_{i 1} a_{(i+1) 1}$, $a_{i 1} a_{(i+1) 2}, a_{i 2} a_{(i+1) 1}$, and $a_{i 2} a_{(i+1) 2}$, for each odd $i$ between 1 and $m-1$ will cover all possibilities. Note that these values need to be computed once for all possible shift 
amounts of the biometrics (since only server's $Y$ 's are shifted). Depending on the distribution of the set bits in each $M(Y)$, the number of modular multiplication now will be between $q_{1} m / 2$ (when $M\left(Y_{i}\right)=M\left(Y_{i+1}\right)$ for each odd $i$ ) and $m\left(q_{0}+\left(1-2 q_{0}\right) / 2\right)=$ $m / 2$ (when $M\left(Y_{i}\right) \neq M\left(Y_{i+1}\right)$ for as many odd $i$ 's as possible). This approach can be also applied to the computation of $d^{j}$ (where only the value of $a_{i 3} a_{(i+1) 3}$ needs to be precomputed for each odd $i$ ) resulting in the same computational savings during computation of the hamming distance. Furthermore, by precomputing the combinations of more than two values additional savings can be achieved during processing of each $Y$.

Optimized Encryption Scheme. As it is clear from the protocol description, its performance crucially relies on the performance of the underlying homomorphic encryption scheme for encryption, addition of two encrypted values, and decryption. Instead of utilizing a general purpose encryption scheme such as Paillier, we turn our attention to schemes of restricted functionality which promise to offer improved efficiency. In particular, the DGK additively homomorphic encryption scheme [13 12] was developed to be used for secure comparison, where each ciphertext encrypts a bit. In that setting, it has faster encryption and decryption time than Paillier and each ciphertext has size $k$ using a $k$-bit RSA modulus (while Paillier ciphertext has size $2 k$ ). To be suitable for our application, the encryption scheme needs to support larger plaintext sizes. The DGK scheme can be modified to work with longer plaintexts. In that case, at decryption time, one needs to additionally solve the discrete logarithm problem where the base is 2-smooth using Pohlig-Hellman algorithm. This means that decryption uses additional $O(n)$ modular multiplications for $n$-bit plaintexts. Now recall that in the protocol we encrypt messages of length $\lceil\log m\rceil+\ell+\kappa$ bits. The use of the security parameter $\kappa$ significantly increases the length of the plaintexts. We, however, notice that the DGK encryption can be setup to permit arithmetic on encrypted values such that all computations on the underlying plaintexts are carried modulo $2^{n}$ for any $n$. For our protocol it implies that (i) the blinding values $r_{S}^{j}$ and $t_{S}^{j}$ can now be chosen from the range $\left[0,2^{n}-1\right]$, where $n=\lceil\log m\rceil+\ell$, and (ii) this provides information-theoretic hiding (thus improving the security properties of the protocol). This observation has a profound impact not only on the client decryption time in step 3(a).iv (which decreases by about an order of magnitude), but also on the consecutive garbled circuit evaluation, where likewise the circuit size is significantly reduced in size.

Circuit Construction. We construct garbled circuits using the most efficient techniques from [35] and references therein. By performing addition modulo $2^{n}$ and eliminating gates which have a constant value as one of their inputs, we reduce the complexity of the circuit for addition to $n-1$ non-XOR gates and $5(n-1)-1$ total gates. Similarly, after eliminating gates with one constant input, the complexity of the circuit for comparison of $n$-bit values becomes $n$ non-XOR gates and $4 n-2$ gates overall. Since in the protocol there are two additions and one comparison per each $j$ followed by $2 c$ OR gates, the size of the overall circuit is $14(n-1)(2 c+1)+2 c$ gates, $(3 n-2)(2 c+1)+2 c$ of which are non-XOR gates. Note that this circuit does not 
Table 1. Breakdown of the performance of the iris identification protocol. Time is expressed in milliseconds unless otherwise stated, and communication overhead in KB.

\begin{tabular}{|c|c|c|c|c||c|c|c|}
\hline \multirow{2}{*}{} & \multirow{2}{*}{ Setup } & \multicolumn{3}{|c||}{ Offline } & \multicolumn{3}{c|}{ Online } \\
\cline { 2 - 8 } & enc & circuit & total & enc & circuit & total \\
\hline \multirow{2}{*}{ erver } & $c=5$ & $1398+71 / \mathrm{rec}$ & $1780+8.5 / \mathrm{rec}$ & $3178+79.5 / \mathrm{rec}$ & $108+148 / \mathrm{rec}$ & $1.2 / \mathrm{rec}$ & $89+149.2 / \mathrm{rec}$ \\
\cline { 2 - 8 } & $c=0$ & $1398+6.5 / \mathrm{rec}$ & $1457+0.7 / \mathrm{rec}$ & $2855+7.2 / \mathrm{rec}$ & $108+13.6 / \mathrm{rec}$ & $0.1 / \mathrm{rec}$ & $89+13.7 / \mathrm{rec}$ \\
\hline \multirow{2}{*}{ Client } & $c=5$ & $11.93 \mathrm{~s}$ & $1693+3.4 / \mathrm{rec}$ & $13.62 \mathrm{~s}+3.4 / \mathrm{rec}$ & $20 / \mathrm{rec}$ & $2.6 / \mathrm{rec}$ & $22.6 / \mathrm{rec}$ \\
\cline { 2 - 8 } & $c=0$ & $11.93 \mathrm{~s}$ & $1055+0.3 / \mathrm{rec}$ & $12.99 \mathrm{~s}+0.3 / \mathrm{rec}$ & $1.8 / \mathrm{rec}$ & $0.2 / \mathrm{rec}$ & $2.0 / \mathrm{rec}$ \\
\hline \multirow{2}{*}{ Comm } & $c=5$ & 512 & $11.6+22.1 / \mathrm{rec}$ & $524+22.1 / \mathrm{rec}$ & $0.5+2.7 / \mathrm{rec}$ & $17.2 / \mathrm{rec}$ & $0.5+19.9 / \mathrm{rec}$ \\
\cline { 2 - 8 } & $c=0$ & 512 & $11.6+2 / \mathrm{rec}$ & $524+2 / \mathrm{rec}$ & $0.5+0.2 / \mathrm{rec}$ & $1.6 / \mathrm{rec}$ & $0.5+1.8 / \mathrm{rec}$ \\
\hline
\end{tabular}

use multiplexers, which are required (and add complexity) during direct computation of minimum.

Oblivious Transfer. The above circuit requires each party to supply $2 n(2 c+1)$ input bits, and a new circuit is used for each $Y$ in $D$. Similar to [18], the combination of techniques from [24] and [32] achieves the best performance in our case. Let the server create each circuit and the client evaluate them. Using the results of [24], performing $O T_{1}^{2}$ the total of $2 n(2 c+1)|D|$ times, where the client receives a $\kappa$-bit string as a result of each OT for a a security parameter $\kappa$, can be reduced to $\kappa$ invocations of $O T_{1}^{2}$ (that communicates to the receiver $\kappa$-bit strings) at the cost of $4 \kappa \cdot 2 n(2 n+1)|D|$ bits of communication and $4 n(2 c+1)$ applications of a hash function for the sender and $2 n(2 c+1)$ applications for the receiver. Then $\kappa O T_{1}^{2}$ protocols can be implemented using the construction of [32] with low amortized complexity, where the sender performs $2+\kappa$ and the receiver performs $2 \kappa$ modular exponentiations with the communication of $2 \kappa^{2}$ bits and $\kappa$ public keys. The OT protocols can be performed during the offline stage, while the additional communication takes place once the inputs are known.

Further Reducing Online Communication. If transmitting $2 m$ ciphertexts during the online stage of the protocol (which amounts to a few hundred KB for our set of parameters) constitutes a burden, this communication can be performed at the offline stage before the protocol begins. This can be achieved using the technique of [33]. We refer the reader to the full version [7] for details of applying this technique to our solution.

\subsection{Implementation and Performance}

We implemented the secure iris identification protocol in C using MIRACL library [22] for cryptographic operations. The implementation used DGK encryption scheme [13 12] with a 1024-bit modulus and another security parameter $t$ set to 160, as suggested in [13 12]. To simplify comparisons with prior work, throughout this work we use $k=1024$ security parameter for public-key cryptography and $\kappa=80$ for symmetric and statistical security. The experiments were run on an Intel Core 2 Duo $2.13 \mathrm{GHz}$ with $3 \mathrm{~GB}$ of RAM and $g c c$ version 4.4.5 on Linux.

Table 1 shows performance of the secure iris identification protocol and its components. The performance was obtained using the following set of parameters: the size of iris code and mask $m=2048$ (this value of $m$ is used in commercial iris recognition 
software), $75 \%$ of bits are reliable in each iris code, and the length $n$ of values is 20 bits. All optimizations described earlier in this section were implemented. In our implementation, upon receipt of client's data, the server precomputes all combinations for pairs of ciphertexts $b_{i} b_{i+1}$ in step 3(a).ii (one-time cost of the total of $4(\mathrm{~m} / 2)$ modular multiplications) and all combinations of 4 elements $d_{i} d_{i+1} d_{i+2} d_{i+3}$ in step 3(a).iii (one-time cost of $11(\mathrm{~m} / 4)$ modular multiplications). This cuts the server's time for processing each $Y$ by more than a half. Furthermore, the constant overhead associated with the OT (circuit) can be reduced in terms of both communication and computation for both parties if public-key operations are implemented over elliptic curves.

The table shows performance using different configurations with the amount of rotation $c=5$ and no rotation with $c=0$ (this is used when the images are well aligned, which is the case for iris biometrics collected at our institution). In the table, we divide the computation and communication into offline precomputation and online protocol execution. No inputs are assumed to be known by any party at precomputation time. Some of the overhead depends on the server's database size, in which case the computation and communication are indicated per record (using notation "/rec"). The overhead associated with the part of the protocol that uses homomorphic encryption is shown separately from the overhead associated with garbled circuits. The offline and online computation for the part based on homomorphic encryption is computed as described in Section 4.2. For circuits, garbled circuit creation, communication, and some of OT is performed at the offline stage, while the rest of OT (as described in Section 4.2) and garbled circuit evaluation takes place during the online protocol execution.

It is evident that our protocol design and optimizations allow us to achieve notable performance. In particular, comparison of two iris codes, which includes computation of $2(2 c+1)=22$ Hamming distances over 2048-bit biometrics in encrypted form, is done in $0.15 \mathrm{sec}$. This is noticeably lower than $0.3 \mathrm{sec}$ online time per record reported by the best currently known face recognition protocol SCiFI [33], which computes a single Hamming distance over 900-bit values. That is, despite an order of magnitude larger number of operations and more complex operations such as division, computation of minimum, etc., we are able to outperform prior work by roughly $50 \%$. This in particular implies that using the techniques suggested in this work (and DGK encryption scheme in particular) performance of SCiFI and other existing protocols can be improved to a fraction of the previously reported time. When iris images are well aligned and no rotation is necessary our protocol requires only $14 \mathrm{msec}$ online computation time and under $2 \mathrm{~KB}$ of data to compare two biometrics.

\section{Secure Fingerprint Identification}

In this section we illustrate how a number of the techniques developed in this work for iris identification can be applied to other types of biometric computations such as FingerCodes. In particular, we show that the efficiency of the secure protocol for FingerCode identification [3] can be improved by an order of magnitude.

The computation involved in FingerCode comparisons is very simple, which results in an extremely efficient privacy-preserving realization. Similar to [3], we rewrite the computation in equation 3 as $\sum_{i=1}^{m}\left(x_{i}-y_{i}\right)^{2}=\sum_{i=1}^{m}\left(x_{i}\right)^{2}+\sum_{i=1}^{m}\left(y_{i}\right)^{2}$ 
Input: $C$ has biometric $X=\left(x_{1}, \ldots, x_{m}\right)$ and DGK encryption key pair $(p k, s k) ; S$ has a database $D$ composed of biometrics $Y=\left(y_{1}, \ldots, y_{m}\right)$.

Output: $C$ learns what records in $D$ resulted in match with $X$ if any, i.e., it learns a bit as a result of comparison of $X$ with each $Y \in D$.

\section{Protocol steps:}

1. $C$ computes and sends to $S$ encryptions $\operatorname{Enc}\left(-2 x_{1}\right), \ldots, \operatorname{Enc}\left(-2 x_{m}\right), \operatorname{Enc}\left(\sum_{i=1}^{m} x_{i}^{2}\right)$.

2. For each $Y=\left(y_{1}, \ldots, y_{m}\right) \in D, S$ and $C$ perform in parallel:

(a) $S$ computes the encrypted distance $d$ between $X$ and $Y$ as $d=\operatorname{Enc}\left(\sum_{i=1}^{m} x_{i}^{2}\right)$. $\operatorname{Enc}\left(\sum_{i=1}^{m} y_{i}^{2}\right) \cdot \prod_{i=1}^{m} \operatorname{Enc}\left(-2 x_{i}\right)^{y_{i}}=\operatorname{Enc}\left(\sum_{i=1}^{m}\left(x_{i}-y_{i}\right)^{2}\right)$, blinds it as $d^{\prime}=$ $d \cdot \operatorname{Enc}\left(r_{S}\right)$, where $r_{S} \stackrel{R}{\longleftarrow}\{0,1\}^{n}$, and sends $d^{\prime}$ to $C$.

(b) $C$ decrypts the value it receives and sets $r_{C}=\operatorname{Dec}\left(d^{\prime}\right)$.

(c) $C$ and $S$ engage in a secure protocol that computes $\left(\left(r_{C}-r_{S}\right) \bmod 2^{n}\right) \stackrel{?}{<} T^{2}$ using garbled circuit evaluation. $S$ creates the circuit and sends it to $C$ along with the keyvalue mapping for the output gate. $C$ obtains keys corresponding to its inputs from $S$ using OT, evaluates the circuit, and learns the result.

Fig. 2. Secure two-party protocol for FingerCode identification

$-\sum_{i=1}^{m} 2 x_{i} y_{i}<T^{2}$. In our protocol, the Euclidean distance is computed using homomorphic encryption, while the comparisons are performed using garbled circuits. The secure FingerCode protocol is given in Figure 2, the client contributes encryptions of $-2 x_{i}$ and $\sum\left(x_{i}\right)^{2}$ to the computation, while the server contributes $\sum\left(y_{i}\right)^{2}$ and computes encryption of $-2 x_{i} y_{i}$ from $-2 x_{i}$. Note that by using $\operatorname{Enc}\left(-2 x_{i}\right)$ instead of $\operatorname{Enc}\left(x_{i}\right)$, the server's work for each $Y$ is reduced since negative values use significantly longer representations. The protocol in Figure 2 uses DGK encryption with the plaintext space of $\left[0,2^{n}-1\right]$. To be able to represent the Euclidean distance, we need to set $n=\lceil\log m\rceil+2 \ell+1$, where $\ell$ is the bitlength of elements $x_{i}$ and $y_{i}$. This implies that all computation on plaintexts is performed modulo $2^{n}$; for instance, $2^{n}-2 x_{i}$ is used in step 1 to form $\operatorname{Enc}\left(-2 x_{i}\right)$. The circuit used in step 2(c) takes two $n$-bit values, adds them modulo $2^{n}$, and compares the result to a constant as described in Section 4.2 .

Finally, some of the computation can be performed offline: for the client it includes precomputing the random values used in the $m+1$ ciphertexts it sends in step 1 (computation of $h^{r} \bmod N$ ), and for the server includes precomputing $\operatorname{Enc}\left(r_{S}\right)$ and preparing a garbled circuit for each $Y$, as well as one-time computation of random values for $\operatorname{Enc}\left(\sum_{i=1}^{m}\left(y_{i}\right)^{2}\right)$ since the reuse of such randomness does not affect security. The client and the server also perform some of OT functionality prior to protocol initiation.

In the FingerCode protocol of [3], each fingerprint in the server's database is represented by $c$ FingerCodes that correspond to different orientations of the same fingerprint, which improves the accuracy of matching. The protocol of [3], however, reports all matches within the $c$ FingerCodes corresponding to the same fingerprint, and this is what our protocol in Figure 2 computes. If it is desirable to output only a single bit for all $c$ instances of a fingerprint, it is easy to modify the circuit evaluated in step 2(c) of the protocol to compute the OR of the bits produced by the original $c$ circuits.

Security. The security of this protocol is straightforward to show and we omit the details of the simulator from the current description. 
Table 2. Breakdown of the performance of the FingerCode identification protocol. Time is expressed in milliseconds and communication overhead in KB.

\begin{tabular}{|c|c|c|c||c|c|c|}
\hline & \multicolumn{3}{|c||}{ Offline } & \multicolumn{3}{c|}{ Online } \\
\cline { 2 - 7 } & enc & circuit & total & enc & circuit & total \\
\hline Server & $3.6+3.9 / \mathrm{rec}$ & $1448+0.37 / \mathrm{rec}$ & $1451.6+4.3 / \mathrm{rec}$ & $0.22+1.37 / \mathrm{rec}$ & $0.05 / \mathrm{rec}$ & $0.22+1.42 / \mathrm{rec}$ \\
\hline Client & 61 & $1025+0.15 / \mathrm{rec}$ & $1086+0.15 / \mathrm{rec}$ & $4.7+0.92 / \mathrm{rec}$ & $0.16 / \mathrm{rec}$ & $4.7+1.08 / \mathrm{rec}$ \\
\hline Comm & 0 & $11.6+1.26 / \mathrm{rec}$ & $11.6+1.26 / \mathrm{rec}$ & $2.12+0.12 / \mathrm{rec}$ & $0.74 / \mathrm{rec}$ & $2.12+0.86 / \mathrm{rec}$ \\
\hline
\end{tabular}

Implementation and Performance. The FingerCode parameters can range as $m=$ $16-640, \ell=4-8$, and $c=5$. We implement the protocol using parameters $m=16$ and $\ell=7$ (the same as in [3]) and therefore $n=19$. The performance of our secure FingerCode identification protocol is given in Table 2. No inputs $(X$ or $Y$ ) are assumed to be known at the offline stage when the parties compute randomization values of the ciphertexts. For that reason, a small fixed cost is inquired in the beginning of the protocol to finish forming the ciphertext using the data itself. We also note that, based on our additional experiments, by using Paillier encryption instead of DGK encryption, the server's online work increases by an order of magnitude, even if packing is used.

It is evident that the overhead reported in the table is minimal and the protocol is well suited for processing fingerprint data in real time. In particular, for a database of 320 records used in prior work (64 fingerprints with 5 FingerCodes each used in [3]), client's online work is $0.35 \mathrm{sec}$ and the server's online work is $0.45 \mathrm{sec}$, with online communication of $279 \mathrm{~KB}$. As can be seen from these results, computation is no longer the bottleneck and this secure two-party protocol can be carried out extremely efficiently. Compared to the solution in [3] that took $16 \mathrm{sec}$ for the online stage with the same setup, the computation speed up is by a factor of 35. Communication efficiency, however, is what was specifically emphasized in the protocol of [3] resulting in $10101 \mathrm{~KB}$ online overhead for a database of size 320 . Our solution therefore improves such result by a factor of 35. We also would like to note that all offline work in [3] is for ciphertext precomputation (since no garbled circuits are used) and is non-interactive, while in our protocol circuit transmission and input-independent portions of OT can be done prior to the protocol itself and involve interaction. We, however, note that the overall (offline and online) computation for $|D|=320$ is $1.48 \mathrm{sec}$ for the client and $3.27 \mathrm{sec}$ for the server with the total of $692 \mathrm{~KB}$ communication, which is still at least several times lower than the online portion of the time and communication in [3].

Privacy-preserving face recognition techniques by Sadeghi et al. [37] can also be adapted to perform secure FingerCode comparisons. They were developed for a different context, but also involve computing Euclidean distances using homomorphic encryption, followed by garbled circuits-based comparisons of the results. (Comparison of face images also includes projecting a client's face to a different vector space as the first step of the protocol, but it is not needed here.) The authors of [37] use Paillier homomorphic encryption for distance computation, where packing of multiple values into a single ciphertext is used at certain points of the protocol. In particular, ciphertext $d^{\prime}$ that the server sends to the client in step 2(b) contains up to $t=\left\lfloor\frac{k-\kappa}{n}\right\rfloor$ distances (for $k$-bit modulus and statistical security parameter $\kappa$ ), where $t=49$ in our case. This results in fewer $\operatorname{Enc}\left(r_{S}\right)$ to form, transmit, and decrypt. 
When we compare communication of our protocol with that based on techniques of [37], we obtain that the initial transmission of client biometric is lower by a factor of 2 in our protocol. The circuit size, and thus corresponding work and communication, is slightly larger in [37] due to handling of additional $\kappa$ bits per $t$ distances. The communication associated with transmission of encrypted blinded distances, however, is significantly lower in [37] due to packing. Overall, we obtain that communication of both solutions is very similar because the communication overhead is heavily dominated by garbled circuits. For the distance computation, [37] report runtime of $6.08 \mathrm{sec}$ for the client and $0.47 \mathrm{sec}$ for the server for $|D|=320$, while distance computation in our protocol (including precomputation) is $0.36 \mathrm{sec}$ for the client and $1.69 \mathrm{sec}$ for the server for the same $|D|$. In [37] the number of dimensions $m=12$, while we have $m=16$, but the length of values is $n=50$ in [37], while we have $n=19$. The computation itself in [37] is more expensive (including interaction between the parties, which we do not have) due to the need to transform client's data, but faster machines are used.

To obtain a better insight on how performance of Paillier encryption with packing compares to that of DGK encryption for our application, we implemented our protocol using techniques of [37] (note that distance computation is more efficient than what is described in [37]). We used a 1024-bit modulus and a number of optimizations suggested in [34] for best performance. In particular, small generator $g=2$ was used to achieve lower encryption time, and decryption is sped up using pre-computation and Chinese remainder computation (see [34], section 7 for more detail). For optimally packed values which result in the lowest overhead per record, we obtain that the server's precomputation is $31.9+1.31 / \mathrm{rec}$ (all in msec), server's online work is $1.0+24.68 / \mathrm{rec}$, client's precomputation is 545.4 , and client's online work is $509.6+$ $0.22 /$ rec. For $|D|=320$, we obtain the client's overall runtime of $1.13 \mathrm{sec}$ and server's runtime of $8.24 \mathrm{sec}$, where the increase in time compared to the performance in [37] can be explained by larger $m$ and slower machines (note that this is the opposite of what is reported in [37]; the server clearly performs the majority of distance computation work). We obtain that our approach is faster by a factor of almost 5 than the use of Paillier encryption with packing as suggested in [37] and online work is faster by a factor of 9.3. And as previously described, the circuit overhead of [37] is slightly larger due to the need to achieve statistical hiding of computed distances.

\section{Conclusions}

The protocol design presented in this work suggests certain principles that lead to an efficient implementation of a privacy-preserving protocol for biometric identification: (i) representation of client's biometric plays an important role; (ii) operations that manipulate bits are the fastest using tools other than encryption; (iii) a proper tuning of encryption tools can result in a significant speedup. Using these principles and a number of new techniques in this work we develop and implement secure protocols for iris and fingerprint identification that use standard biometric recognition algorithms. The optimization techniques employed in this work allow us to achieve notable performance results for different secure biometric identification protocols.

In particular, we develop the first privacy-preserving two-party protocol for iris codes using current biometric recognition algorithms. Despite the length of iris codes' 
representation and the complexity of their processing, our protocol allows a secure comparison between two biometrics to be performed in $0.15 \mathrm{sec}$ with communication of under $18 \mathrm{~KB}$. Furthermore, when the iris codes are known to be well-aligned and their rotation is not necessary, the overhead decreases by an order of magnitude to $14 \mathrm{msec}$ computation and $2 \mathrm{~KB}$ communication per comparison.

Two FingerCodes used for fingerprint recognition can be compared at low cost, which allowed us to develop an extremely efficient privacy-preserving protocol. Comparing two fingerprints requires approximately $1 \mathrm{msec}$ of computation, allowing thousands of biometrics to be processed in a matter of seconds. Communication overhead is also very modest with less than $1 \mathrm{~KB}$ per biometric comparison. Compared to prior privacy-preserving implementation of FingerCode [3], we simultaneously improve online computation and communication by a factor of more than 30 .

Acknowledgments. We would like to thank Keith Frikken for suggestion to reduce communication of the iris identification protocol from $3 m$ to $2 m$ ciphertexts. We would also like to thank Stefan Katzenbeisser and the anonymous reviewers for their insightful comments and observations. Portions of this work were sponsored by the Air Force Office of Scientific Research grant AFOSR-FA9550-09-1-0223.

\section{References}

1. Atallah, M., Bykova, M., Li, J., Frikken, K., Topkara, M.: Private collaborative forecasting and benchmarking. In: ACM Workshop on Privacy in the Electronic Society (WPES), pp. 103-114 (2004)

2. Barbosa, M., Brouard, T., Cauchie, S., de Sousa, S.M.: Secure biometric authentication with improved accuracy. In: Mu, Y., Susilo, W., Seberry, J. (eds.) ACISP 2008. LNCS, vol. 5107, pp. 21-36. Springer, Heidelberg (2008)

3. Barni, M., Bianchi, T., Catalano, D., Di Raimondo, M., Labati, R., Failla, P., Fiore, D., Lazzeretti, R., Piuri, V., Scotti, F., Piva, A.: Privacy-preserving fingercode authentication. In: ACM Workshop on Multimedia and Security (MM\&Sec), pp. 231-240 (2010)

4. Barzegar, N., Moin, M.: A new user dependent iris recognition system based on an area preserving pointwise level set segmentation approach. EURASIP Journal on Advances in Signal Processing, 1-13 (2009)

5. Blanton, M.: Empirical evaluation of secure two-party computation models. Technical Report TR 2005-58, CERIAS, Purdue University (2005)

6. Blanton, M., Aliasgari, M.: Secure computation of biometric matching. Technical Report 2009-03, Department of Computer Science and Engineering, University of Notre Dame (2009)

7. Blanton, M., Gasti, P.: Secure and Efficient Protocols for Iris and Fingerprint Identification. Cryptology ePrint Archive, Report 2010/627 (2010), http: / / eprint.iacr.org/

8. Bringer, J., Chabanne, H., Izabachène, M., Pointcheval, D., Tang, Q., Zimmer, S.: An Application of the Goldwasser-Micali Cryptosystem to Biometric Authentication. In: Pieprzyk, J., Ghodosi, H., Dawson, E. (eds.) ACISP 2007. LNCS, vol. 4586, pp. 96-106. Springer, Heidelberg (2007)

9. Bunn, P., Ostrovsky, R.: Secure two-party k-means clustering. In: ACM Conference on Computer and Communications Security (CCS), pp. 486-497 (2007)

10. Catrina, O., Saxena, A.: Secure computation with fixed-point numbers. In: Sion, R. (ed.) FC 2010. LNCS, vol. 6052, pp. 35-50. Springer, Heidelberg (2010) 
11. Cramer, R., Damgård, I.B., Nielsen, J.B.: Multiparty computation from threshold homomorphic encryption. In: Pfitzmann, B. (ed.) EUROCRYPT 2001. LNCS, vol. 2045, pp. 280-300. Springer, Heidelberg (2001)

12. Damgård, I., Geisler, M., Krøigård, M.: A correction to efficient and secure comparison for on-line auctions. Cryptology ePrint Archive, Report 2008/321 (2008)

13. Damgård, I., Geisler, M., Krøigård, M.: Homomorphic encryption and secure comparison. Journal of Applied Cryptology 1(1), 22-31 (2008)

14. Damgård, I., Geisler, M., Krøigaard, M., Nielsen, J.B.: Asynchronous multiparty computation: Theory and implementation. In: Jarecki, S., Tsudik, G. (eds.) PKC 2009. LNCS, vol. 5443, pp. 160-179. Springer, Heidelberg (2009)

15. Daugman, J.: How iris recognition works. IEEE Transactions on Circuits and Systems for Video Technology 14(1), 21-30 (2004)

16. Erkin, Z., Franz, M., Guajardo, J., Katzenbeisser, S., Lagendijk, I., Toft, T.: Privacypreserving face recognition. In: Goldberg, I., Atallah, M.J. (eds.) PETS 2009. LNCS, vol. 5672, pp. 235-253. Springer, Heidelberg (2009)

17. Frikken, K.B.: Privacy-preserving set union. In: Katz, J., Yung, M. (eds.) ACNS 2007. LNCS, vol. 4521, pp. 237-252. Springer, Heidelberg (2007)

18. Henecka, W., Kogl, S., Sadeghi, A.-R., Schneider, T., Wehrenberg, I.: TASTY: Tool for Automating Secure Two-partY computations. In: ACM Conference on Computer and Communications Security (CCS), pp. 451-462 (2010)

19. Hoens, T., Blanton, M., Chawla, N.: A private and reliable recommendation system using a social network. In: IEEE International Conference on Information Privacy, Security, Risk and Trust (PASSAT), pp. 816-825 (2010)

20. Hollingsworth, K., Bowyer, K., Flynn, P.: The best bits in an iris code. IEEE Transactions on Pattern Analysis and Machine Intelligence 31(6), 964-973 (2009)

21. U.S. DHS US-VISIT, http://www. dhs.gov/files/programs/usv.shtm

22. Multiprecision Integer and Rational Arithmetic C/C++ Library, http: //www. shamus.ie/

23. IrisGuard Press Release, http://cl.1y/3KIB

24. Ishai, Y., Kilian, J., Nissim, K., Petrank, E.: Extending oblivious transfers efficiently. In: Boneh, D. (ed.) CRYPTO 2003. LNCS, vol. 2729, pp. 145-161. Springer, Heidelberg (2003)

25. Jain, A., Prabhakar, S., Hong, L., Pankanti, S.: Filterbank-based fingerprint matching. IEEE Transactions on Image Processing 9(5), 846-859 (2000)

26. Kolesnikov, V., Sadeghi, A.-R., Schneider, T.: Improved garbled circuit building blocks and applications to auctions and computing minima. In: Garay, J.A., Miyaji, A., Otsuka, A. (eds.) CANS 2009. LNCS, vol. 5888, pp. 1-20. Springer, Heidelberg (2009)

27. Kolesnikov, V., Schneider, T.: Improved garbled circuit: Free XOR gates and applications. In: Aceto, L., Damgård, I., Goldberg, L.A., Halldórsson, M.M., Ingólfsdóttir, A., Walukiewicz, I. (eds.) ICALP 2008, Part II. LNCS, vol. 5126, pp. 486-498. Springer, Heidelberg (2008)

28. Lindell, Y., Pinkas, B., Smart, N.P.: Implementing two-party computation efficiently with security against malicious adversaries. In: Ostrovsky, R., De Prisco, R., Visconti, I. (eds.) SCN 2008. LNCS, vol. 5229, pp. 2-20. Springer, Heidelberg (2008)

29. Freedman, M.J., Nissim, K., Pinkas, B.: Efficient private matching and set intersection. In: Cachin, C., Camenisch, J.L. (eds.) EUROCRYPT 2004. LNCS, vol. 3027, pp. 1-19. Springer, Heidelberg (2004)

30. Malkhi, D., Nisan, N., Pinkas, B., Sella, Y.: Fairplay - a secure two-party computation system. In: USENIX Security Symposium, pp. 287-302 (2004)

31. Maltoni, D., Maio, D., Jain, A., Prabhakar, S.: Hanbook of Fingerprint Recognition, 2nd edn. Springer, Heidelberg (2009)

32. Naor, M., Pinkas, B.: Efficient oblivious transfer protocols. In: ACM-SIAM Symposium on Discrete Algorithms (SODA), pp. 448-457 (2001) 
33. Osadchy, M., Pinkas, B., Jarrous, A., Moskovich, B.: SCiFI - A system for secure face identification. In: IEEE Symposium on Security and Privacy, pp. 239-254 (2010)

34. Paillier, P.: Public-key cryptosystems based on composite degree residuosity classes. In: Stern, J. (ed.) EUROCRYPT 1999. LNCS, vol. 1592, pp. 223-238. Springer, Heidelberg (1999)

35. Pinkas, B., Schneider, T., Smart, N.P., Williams, S.C.: Secure two-party computation is practical. In: Matsui, M. (ed.) ASIACRYPT 2009. LNCS, vol. 5912, pp. 250-267. Springer, Heidelberg (2009)

36. Rabin, T., Ben-Or, M.: Verifiable secret sharing and multiparty protocols with honest majority. In: ACM Symposium on Theory of Computing (STOC), pp. 73-85 (1989)

37. Sadeghi, A.-R., Schneider, T., Wehrenberg, I.: Efficient privacy-preserving face recognition. In: Lee, D., Hong, S. (eds.) ICISC 2009. LNCS, vol. 5984, pp. 229-244. Springer, Heidelberg (2010)

38. Troncoso-Pastoriza, J., Katzenbeisser, S., Celik, M.: Privacy preserving error resilient DNA searching through oblivious automata. In: ACM Conference on Computer and Communications Security (CCS), pp. 519-528 (2007)

39. Yao, A.: How to generate and exchange secrets. In: IEEE Symposium on Foundations of Computer Science (FOCS), pp. 162-167 (1986)

\section{A Security Analysis of the Iris Protocol}

Security of the iris protocol relies on the security of the underlying building blocks. In particular, we need to assume that (i) the DGK encryption scheme is semantically secure (which was shown under a hardness assumption that uses subgroups of an RSA modulus [13,12]); (ii) garbled circuit evaluation is secure (which was shown assuming that the hash function is correlation robust [27], or if it is modeled as a random oracle); and (iii) the oblivious transfer is secure as well (to achieve this, techniques of [24] require the hash function to be correlation robust and the use of a pseudo-random number generator, while techniques of [32] model the hash functions as a random oracle and use the computational Diffie-Hellman (CDH) assumption). Therefore, assuming the security of the DGK encryption, $\mathrm{CDH}$, and using the random oracle model for hash functions is sufficient for our solution.

To show the security of the protocol, we sketch how to simulate the view of each party using its inputs and outputs alone. If such simulation is indistinguishable from the real execution of the protocol, for semi-honest parties this implies that the protocol does not reveal any unintended information to the participants (i.e., they learn only the output and what can be deduced from their respective inputs and outputs).

First, consider the client $C$. The client's input consists of its biometric $X, M(X)$ and the private key, and its outputs consists of a bit $b$ for each record in $S$ 's database $D$. A simulator that is given these values simulates $C$ 's view by sending encrypted bits of $C$ 's input to the server as prescribed in step 1 of the protocol. It then simulates the messages received by the client in step 3(a).iii using encryptions of two randomly chosen strings $r_{C}^{j}$ and $t_{C}^{j}$ of length $n$. The simulator next creates a garbled circuit for the computation given in step 3(b) that, on input client's $r_{C}^{j}$ 's and $t_{C}^{j}$ 's computes bit $b$, sends the circuit to the client, and simulates the OT. It is clear that given secure implementation of garbled circuit evaluation in the real protocol, the client cannot distinguish simulation from real protocol execution. Furthermore, the values that $C$ recovers in step 3(a).iv of the 
protocol are distributed identically to the values used in the real protocol execution that uses DGK encryption (and they are statistically indistinguishable when other encryption schemes are used).

Now consider the server's view. The server has its database $D$ consisting of $Y, M(Y)$ and the threshold $T$ as the input and no output. In this case, a simulator with access to $D$ first sends to $S$ ciphertexts (as in step 1 of the protocol) that encrypt bits of its choice. For each $Y \in D, S$ performs its computation in step 3(a) of the protocol and forms garbled circuits as specified in step 3(b). The server and the simulator engage in the OT protocol, where the simulator uses arbitrary bits as its input to the OT protocol and the server sends the key-value mapping for the output gate. It is clear that the server cannot distinguish the above interaction from the real protocol execution. In particular, due to semantic security of the encryption scheme $S$ learns no information about the encrypted values and due to security of OT $S$ learns no information about the values chosen by the simulator for the garbled circuit. 